\title{
Characterization of the collagen component of cartilage repair tissue of the talus with quantitative MRI: comparison of T2 relaxation time measurements with a diffusion-weighted double-echo steady-state sequence (dwDESS)
}

\author{
M. Kretzschmar • O. Bieri • M. Miska • M. Wiewiorski • \\ N. Hainc $\cdot$ V. Valderrabano $\cdot$ U. Studler \\ Received: 31 March 2014 / Revised: 17 October 2014 / Accepted: 5 November 2014 / Published online: 19 November 2014 \\ (C) European Society of Radiology 2014
}

\begin{abstract}
Objectives The purpose of this study was to characterize the collagen component of repair tissue (RT) of the talus after autologous matrix-induced chondrogenesis (AMIC) using quantitative $\mathrm{T} 2$ and diffusion-weighted imaging.

Methods Mean T2 values and diffusion coefficients of AMIC-RT and normal cartilage of the talus of 25 patients with posttraumatic osteochondral lesions and AMIC repair were compared in a cross-sectional design using partially spoiled steady-state free precession (pSSFP) for T2 quantification, and diffusion-weighted double-echo steady-state (dwDESS) for diffusion measurement. RT and cartilage were graded with modified Noyes and MOCART scores on morphological sequences. An association between follow-up interval and quantitative MRI measures was assessed using multivariate regression, after stratifying the cohort according to time interval between surgery and MRI.

Results Mean T2 of the AMIC-RT and cartilage were $43.1 \mathrm{~ms}$ and $39.1 \mathrm{~ms}$, respectively $(p=0.26)$. Mean diffusivity of the RT $\left(1.76 \mu \mathrm{m}^{2} / \mathrm{ms}\right)$ was significantly higher compared to normal cartilage $\left(1.46 \mu \mathrm{m}^{2} / \mathrm{ms}\right)(p=0.0092)$. No correlation was
\end{abstract}

\footnotetext{
M. Kretzschmar $(\varangle) \cdot$ N. Hainc $\cdot$ U. Studler

Department of Radiology, University Hospital Basel, Petersgraben 4, 4031 Basel, Switzerland

e-mail: martin.kretzschmar@ucsf.edu

O. Bieri

Division of Radiological Physics, University Hospital Basel,

Petersgraben 4, 4031 Basel, Switzerland

M. Miska

Department of Orthopedics, University Hospital, Heidelberg, Germany

M. Wiewiorski $\cdot$ V. Valderrabano

Department of Orthopedic Surgery, University Hospital Basel,

Petersgraben 4, 4031 Basel, Switzerland

found between morphological and quantitative parameters. RT diffusivity was lowest in the subgroup with follow-up $>28$ months $(p=0.027)$.

Conclusions Compared to T2-mapping, dwDESS demonstrated greater sensitivity in detecting differences in the collagen matrix between AMIC-RT and cartilage. Decreased diffusivity in patients with longer follow-up times may indicate an increased matrix organization of RT.

Key Points

- MRI is used to assess morphology of the repair tissue during follow-up.

- Quantitative MRI allows an estimation of biochemical properties of the repair tissue.

- Differences between repair tissue and cartilage were more significant with $d w D E S S$ than T2 mapping.

Keywords Quantitative MRI · DWI · T2 mapping · Cartilage $\cdot$ Chondral repair

\section{Introduction}

Osteochondral lesions (OCLs) of the talus are primarily of traumatic aetiology and often occur in young adults with a history of sports injuries. If untreated, these lesions may result in early osteoarthritis of the ankle joint, with concomitant chronic pain and severely reduced physical activity. Various surgical procedures have been developed for the treatment and reconstruction of osteochondral lesions [1]. In this study, patients were treated with autologous matrix-induced chondrogenesis (AMIC) [2-4]. With this recently introduced method, the lesion is first debrided and microfractured. The resulting blood clot containing progenitor cells, including stem cells and growth factors, is then covered with a commercially available acellular collagen I/III matrix [5]. There is 
evidence that these stem cells subsequently differentiate into chondrocytes in vivo and form a repair tissue that resembles fibrocartilage $[6,7]$. Glycosaminoglycan and collagen are the main components and the two most important constituents of the biomechanical properties of cartilage tissue. Both can be measured in vivo with quantitative MRI, and therefore allow characterization of cartilage repair tissue. This work focuses on the characterization of the collagen component.

Changes in the chondral extracellular matrix such as disorganization and breakdown of the collagen network can be assessed by changes in the apparent transverse relaxation time (T2). Disintegration of the collagen network leads to an increase in the mobility of cartilage water, thus elongating T2. This effect on T2 is independent from GAG concentration [8-10]. Previous in vivo studies have demonstrated elevated $\mathrm{T} 2$ values in patients with osteoarthritis (OA) compared to healthy subjects [11-14]. Several studies have used this parameter to characterize RT after chondral repair in the knee and ankle $[15,16]$.

Moreover, the collagen fibre network shows an ordered architecture, with a predominantly oblique orientation of fibres in the transitional zone $[17,18]$. Overall, the microstructure leads to a reduction of the mean diffusivity of water protons within the cartilage as compared to fluids [19, 20]. A reduced order of the fibre network, based either on degenerative processes in cartilage or incomplete differentiation of RT, leads to an increased diffusivity of water protons, which can be traced using diffusion-weighted sequences [21, 22].

The aim of this study was to evaluate and compare two quantitative MR sequences in measuring the integrity of the collagen fibre component of AMIC-RT of the talus. For this purpose, we applied two recently proposed quantitative imaging techniques in the ankle joint: a partially spoiled steadystate free precession sequence for quantitative T2 mapping and a truly diffusion-weighted double-echo steady-state sequence for quantitative diffusion mapping.

\section{Methods}

Patients

The study was approved by the institutional review board, and written informed patient consent was obtained from all participants. Twenty-six patients who had undergone AMIC cartilage repair after posttraumatic OCL at the talus were enrolled in the study. Exclusion criteria were advanced radiographic osteoarthritis, known inflammatory joint disease such as rheumatoid arthritis, concomitant tibial OCL, contraindications for MRI such as claustrophobia, medical implants that were incompatible with an MRI examination, or technically insufficient MRI examinations. One patient had to be excluded from the MRI analysis because of post-surgical metal artefacts located at the AMIC-RT. The remaining 25 patients had a mean age of 38 years (range 18-56 years) and a mean BMI of 28 (range 19-39). Two-thirds of the cohort ( $n=17$, $68 \%$ ) were men; eight subjects $(32 \%)$ were women. All patients had post-traumatic OCL of the talus at the right $(n=11,44 \%)$ or left side $(n=14,56 \%)$. Eleven patients (44\%) had prior surgery such as retrograde drilling or microfracturing at the site of the OCL. The majority of lesions ( $n=21,84 \%$ ) were located at the medial talar dome. The remaining lesions $(n=4,16 \%)$ were located at the lateral talar dome.

All 25 patients underwent AMIC-aided repair of an OCL of the talus between 2008 and 2010. The operative technique used in the AMIC procedure has been described elsewhere [3, 5]. In brief, depending upon the position of the OCL, a standard anteromedial or anterolateral arthrotomy was performed. In the majority of cases, an additional malleolar osteotomy was needed to gain access to the talar OCL. After debridement of defective cartilage and necrotic bone, and micro-drilling of the underlying osseous component of the lesion, the acellular collagen I/III membrane (Chondro-Gide, Geistlich Pharma AG, Wolhusen, Switzerland) was fixated on the debrided lesion with fibrin glue (Tissucol, Baxter International, Deerfield, IL, USA). At the time of follow-up MRI, 19 patients showed a reduction in pain of $>50 \%$ on a visual analogue scale, five patients showed a reduction in pain of $<50 \%$, and one patient reported aggravated pain. Postoperative care was standardized and consisted of immobilization for the first six weeks, complemented by functional physiotherapy with $15 \mathrm{~kg}$ partial weight-bearing. The patients underwent a 6- to 12-week rehabilitation protocol, including muscle training and proprioceptive training to enhance ankle joint stability under increasing load, with progression to full weight.

\section{MRI}

MRI was obtained with a $3 \mathrm{~T}$ system (MAGNETOM Verio, Siemens AG, Erlangen, Germany) using a 4-channel flexible multipurpose coil (Siemens AG). To reduce involuntary movement and thus avoid artefacts due to mis-registration of data, the foot was placed in a purpose-built mount and fixed with a vacuum mattress. The following $3 \mathrm{D}$ sequences were acquired with identical resolution parameters, with a voxel size of $0.4 \times 0.4 \times 3.0 \mathrm{~mm}^{3}$ and field of view (FOV) of $140 \times 140 \mathrm{~mm}^{2}$.

1. Quantitative T2 mapping: sagittal partially spoiled steadystate free precession (pSSFP) [23]. Two scans were acquired with the following parameters: flip angle $35^{\circ}$; TR $10.09 \mathrm{~ms}$; TE $5.05 \mathrm{~ms}$; partial RF spoiling, phi $=1^{\circ}, 10^{\circ}$; acquisition time, $366 \mathrm{~s}$. 
2. Quantitative diffusion imaging: a sagittal double-echo steady-state (dwDESS) technique was used [24]. Two scans with and without a diffusion-sensitizing gradient were performed. TR, $16.87 \mathrm{~ms}$; TE1, $4.3 \mathrm{~ms}$; TE2 $4.3 \mathrm{~ms}$; diff movement: $150 \mathrm{mT} / \mathrm{m}^{*} \mathrm{~ms}$. flip angle $15^{\circ}$; acquisition time, $370 \mathrm{~s}$.

3. Anatomic imaging: sagittal $3 \mathrm{D}$ dual-echo steady-state (DESS) imaging sequence with repetition time (TR) of $16.87 \mathrm{~ms}$ and echo time (TE) of $4.3 \mathrm{~ms}$.

4. As a second anatomic imaging sequence, we included a coronal proton density-weighted TSE with variable flip angle (SPACE), with TR of $1,300 \mathrm{~ms}$, TE of $37 \mathrm{~ms}$, and voxel size of $0.25 \times 0.25 \times 0.6 \mathrm{~mm}$.

The mean follow-up interval between surgery and MRI was 23 months (range 11-49) months.

Image analysis

Imaging data were analysed in consensus by two experienced, board-certified musculoskeletal radiologists on an OsiriX v. 3.8.1 workstation. Localization and measurement of the RT and morphology of the reference cartilage of the talus were analysed on the anatomic DESS and SPACE sequences.

The morphology of RT was visually assessed using a modified MOCART score with the following RT sub-scores: defect filling, integration of border zone, surface, structure, and signal intensity [25]. Defect filling was graded as $4=$ complete, $3=$ complete with hypertrophy, $2=$ incomplete ( $>50 \%$ of surrounding cartilage), and $1=$ incomplete $(<50 \%$ of surrounding cartilage). Integration of the border zone was graded as $4=$ complete, $3=$ incomplete with slit-like demarcation of the border, $2=$ incomplete with defect $<50 \%$ of lesion length, and $1=$ incomplete with defect $>50 \%$ of lesion length. RT surface was graded as $3=$ intact, $2=$ damage $<50 \%$ of depth, and $1=$ damage $>50 \%$ of depth. RT structure was graded as $2=$ homogeneous and $1=$ heterogeneous. RT signal intensity was graded as $3=$ normal (isointense compared to cartilage), $2=$ nearly normal, and $1=$ abnormal). Finally, the sum of all sub-scores was calculated (RT sum score, maximum sum 16). The size of the RT was measured on coronal and sagittal images using the largest diameters on the level of the cartilage layer. The thickness of the RT was measured in coronal images from the articular surface to the RT bone interface. The cartilage of four ankle regions (medial and lateral talus and medial and lateral tibia) was graded with a modified version of the Noyes score [26] that was adapted to account for the small thickness of the ankle cartilage, with $0=$ normal thickness and signal intensity, $1=$ normal thickness with abnormal signal on fluid-sensitive sequences, $2=$ partial-thickness focal defect involving not more than $50 \%$ of the cartilage thickness, and $3=$ focal cartilage defect deeper than $50 \%$ cartilage thickness, including full-thickness lesions extending to the subchondral bone.

Under the control of coronal images, regions of interest (ROIs) were positioned in the sagittal anatomic DESS sequence within the RT (Fig. 1a) and in the morphologically normal-appearing cartilage layer of the non-affected talar dome of the same joint (Fig. 1b), and then copied to the sagittal quantitative maps. The reference cartilage had to show normal signal characteristics and morphological patterns on the DESS images, and the spatial orientation of the layer within the magnet field had to be similar compared to the zone of RT in order to avoid artificial signal alterations due to the magic angle effect. The ROIs were similar in size and shape.

Statistical analysis

Descriptive statistics were used to analyze the RT characteristics according to MOCART variables and the distribution and prevalence of cartilage lesions.

For the primary outcome, a comparison of quantitative MRI values of AMIC-RT and reference cartilage was performed using the Wilcoxon signed-rank test, given the nonnormal distribution of data. The level of significance was set to $P \leq 0.05$.

In addition, we used linear regression analysis to find correlations between morphological parameters such as cartilage lesions and MOCART RT sum score, and the quantitative MRI measures T2 and diffusion coefficients, respectively. To analyse the association of RT diffusivity and $\mathrm{T} 2$ relaxation time with follow-up time, the patient cohort was stratified in tertiles of follow-up interval, with eight patients in the first tertile ranging from 11-14 months, nine patients in the second tertile ranging from 15-25 months, and eight patients in the third tertile ranging from $28-49$ months. We then used multivariate regression analysis, with diffusion coefficient and $\mathrm{T} 2$ values as outcome measures and follow-up tertiles, and lesion score of the opposing tibial cartilage and RT size as covariates.

For statistical analysis, the Prism 4 (Macintosh) software (GraphPad Software, Inc., San Diego, CA, USA) and JMP version 11 (SAS Institute, Cary, NC, USA) were used.

\section{Results}

Morphological findings of cartilage and repair tissue

The morphological analysis of the SPACE and DESS sequences revealed focal lesions in the talar (two grade 2 lesions, total $n=$ two lesions) and tibial cartilage (two grade 1 and three grade 2 lesions, total $n=$ five lesions) of the non-affected reference compartment. A higher prevalence of focal cartilage 
Fig. 1 DESS sequence for anatomical imaging of the ankle joint cartilage. ROIs with similar size, shape, and spatial orientation towards the magnet field were positioned within the RT (a) and within the normally structured cartilage layer of the talus (b)
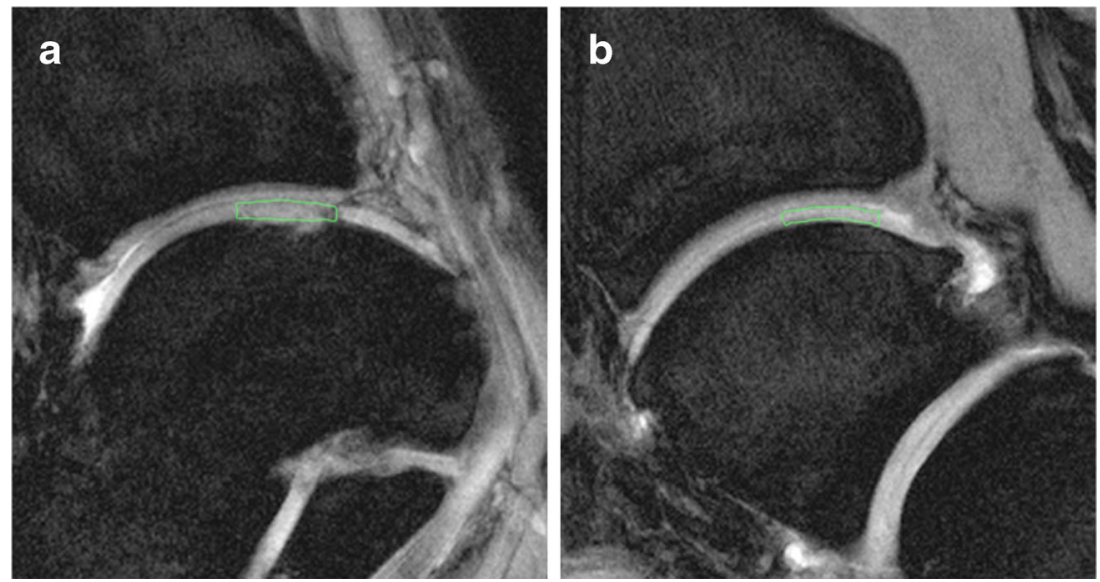

lesions was found in the tibial cartilage of the compartment with the OCL (two grade 1 and six grade 2 lesions, total $n=$ eight lesions). Mean RT size was $1.1 \mathrm{~cm}^{2}$, ranging from 0.5 to $2.2 \mathrm{~cm}^{2}$. Mean thickness of the RT was $1.9 \mathrm{~mm}$, ranging from 0.5 to $4.4 \mathrm{~mm}$. Mean MOCART sub-scores of the RT were as follows: filling, 3.4 (SD 0.9); integration, 2.6 (SD 0.7); surface, 2.3 (0.7); structure, 1.4 (SD 0.5); and signal intensity 1.7 (SD 0.6). The mean sum of all subscores (RT sum score) was 11.4 (SD 2.6).

T2 measurements and diffusion coefficients of reference cartilage and repair tissue

The quantitative measurements of the apparent cartilage water proton diffusivity showed a mean diffusion coefficient of $1.76 \mathrm{\mu m}^{2} / \mathrm{ms}$ (SD 0.68 ) for the RT and $1.46 \mu \mathrm{m}^{2} / \mathrm{ms}$ (SD $0.64)$ for the reference cartilage (Table 1, Figs. 2a and 3). This difference was statistically significant $(\mathrm{p}=0.0092)$. The cartilage of the talus was too thin to reliably perform a laminar analysis of the cartilage T2 that differentiates between a deep and superficial zone. Thus we used ROIs that included both zones and measured the global apparent mean T2. In our cohort, we found a mean T2 value of $43.1 \mathrm{~ms}$ (SD 14.4) for the AMIC-RT and $39.1 \mathrm{~ms}$ (SD 11.5) for the normal cartilage (Table 1, Figs. 2b and Fig. 3). This difference was not statistically significant $(p=0.26)$.

Table $1 \mathrm{~T} 1$ values, T2 values, and Diffusion coefficients obtained from repair tissue and reference cartilage

\begin{tabular}{llll}
\hline Sequence & $\begin{array}{l}\text { AMIC Repair } \\
\text { Tissue }\end{array}$ & $\begin{array}{l}\text { Reference } \\
\text { Cartilage }\end{array}$ & P -value* \\
\hline $\mathrm{T} 2(\mathrm{~ms})$, mean, $(\mathrm{SD})$ & $43.1( \pm 14.4)$ & $39.1( \pm 11.5)$ & 0.26 \\
$\begin{array}{c}\mathrm{dwDESS}\left(\mu \mathrm{m}^{2} / \mathrm{ms}\right), \\
\text { mean }(\mathrm{SD})\end{array}$ & $1.76( \pm 0.68)$ & $1.46( \pm 0.64)$ & 0.0092 \\
\hline
\end{tabular}

*P values calculated with the Wilcoxon signed rank test
Correlation of morphological and quantitative MRI measures

No correlation was found between the cartilage scores of the non-affected talar dome and cartilage diffusion coefficients $\left(r_{\mathrm{s}}=0.03, p=0.926\right)$ or T2 values $\left(r_{\mathrm{s}}=0.245, p=0.261\right)$. No associations were found between the score for the tibial cartilage opposing the OCL and the T2 or diffusion measures of the RT. Comparison of the RT sum score of the MOCART grading system with RT diffusion coefficients revealed no correlation $\left(\mathrm{r}_{\mathrm{s}}-1.6, p=0.471\right)$. There was also no association between the RT sum score and RT T2 values $\left(r_{\mathrm{s}}=-0.29, p=0.172\right)$.

\section{Quantitative MR measures and follow-up time}

Controlling for lesion size and WORMS lesions of the opposing tibial cartilage, in a multivariate linear regression model, we found significantly lower diffusion coefficients in the last follow-up tertile (follow-up intervalls ranging from 28 to 49 months) compared to the first follow-up tertile (follow-up intervalls $11-14$ months) $(p=0.027)$, and by trend, a reduced diffusion coefficient of the second follow-up tertile compared to the first ( $p=0.055$ ) (Fig. 4). No significant differences among follow-up tertiles were found using the same model with $\mathrm{T} 2$ values as outcome variable.

\section{Discussion}

The results of our study show that, compared to quantitative T2 measurement, the diffusion-weighted DESS sequence was more sensitive in detecting differences between RT of AMICtreated OCLs and normal-appearing cartilage. Diffusion coefficients of the RT decreased with follow-up time, reaching significance after 28 months. No such association was found for T2 measures. We found no association between quantitative MRI measures and the MOCART RT scores. 


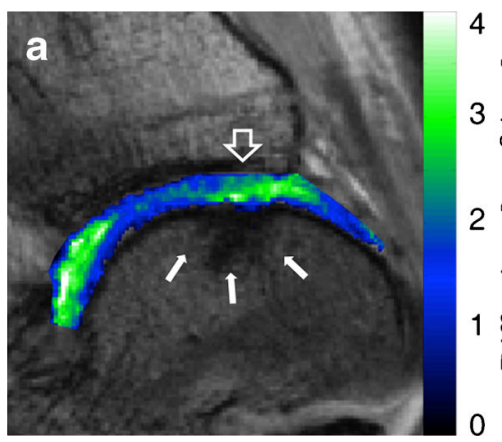

Fig. 2 Fusion of parameter maps with a T1w anatomic sequence of one patient. (a) AMIC-treated OCL of the talus with sclerotic bone marrow changes below the chondral repair (white arrows). The increased diffusity

Compared to the number of studies of the knee, there are relatively few studies focusing on the biochemical characterization of cartilage in the ankle joint. One reason is that the cartilage layer of the talus is thinner [27], thus requiring scans with higher spatial resolution. This problem is further aggravated if quantitative imaging sequences are used. With the increased signal-to-noise ratio and spatial resolution of $3 \mathrm{~T}$ MRI scanners, biochemical imaging of the ankle cartilage has become feasible $[28,29]$.

In this study, we applied - for the first time at the ankle - a recently introduced method for diffusion measurement of the cartilage based on a double-echo steady-state sequence, as described by Bieri et al. [24]. Conventional DWI sequences are based on a single-shot echo-planar imaging technique that provides poor spatial resolution and is used mainly in brain and whole-body imaging. More feasible for musculoskeletal applications such as the evaluation of cartilage are sequences based on a steady-state free-precession technique offering a higher resolution. Instead of a SSFP sequence that was used in the work of Quirbach [28], we used a truly diffusion-weighted $3 \mathrm{D}$ double-echo steady-state (dwDESS) sequence. The great advantage of the dwDESS technique is the independence of the diffusion coefficient with regard to $\mathrm{T} 1$ and $\mathrm{T} 2$ relaxation values, thereby allowing the calculation of absolute diffusion values. This independence may enhance the sensitivity for differences in diffusivity of tissue protons. The sequence we used for T2 mapping was based on a pSSFP technique. The

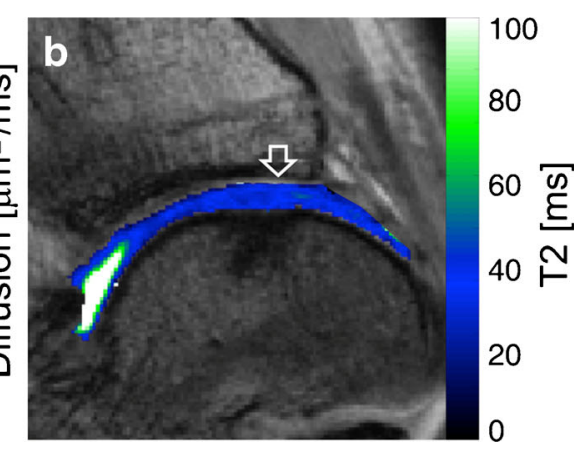

demarcates the RT from the surrounding cartilage (open arrow). (b) The RT shows T2 relaxation times equal to the surrounding normal cartilage (open arrow)

$\mathrm{T} 2$ values we gained for both RT and control cartilage ( $39 \mathrm{~ms}$ and $43 \mathrm{~ms}$ ) were lower compared to the data reported by Quirbach (48 ms and $50 \mathrm{~ms})$. The shorter values are most likely explained by the technical properties of the pSSFP sequence [23]. Since this effect appeared in a systematic way, it should not have influenced the detection of possible differences between RT and control cartilage.

The overall finding that the diffusion-weighted sequence was more sensitive in detecting differences between RT and cartilage versus T2 mapping has also been reported in other studies. Quirsbach et al. [28] utilized 3 T MRI to examine 10 volunteers and 12 patients with matrix-associated autologous chondrocyte transplantation (MACT) at the talus, and found significant differences in diffusivity between the MACT-RT and healthy cartilage, whereas $\mathrm{T} 2$ and $\mathrm{T} 2 *$ did not show significant results. Apprich et al., who compared the diffusion values of MACT and microfracture therapy (MFX) of the talus [30], found that while there was no difference in diffusion quotients between reference cartilage and MACT-RT, significantly higher diffusion quotients were found in the MFX-RT. Another study compared MACT and MFX in patients with chondral repair of the knee [16], reporting significantly higher diffusion values in both MFX- and MACT-RT compared to normal cartilage, whereas lower T2 values were seen only in the MFX-RT compared to normal cartilage. The lower T2 values may be partially explained by histological differences of the RT in the techniques: MFX-RT resembles the qualities

Fig. 3 Box and whisker plots of

DW

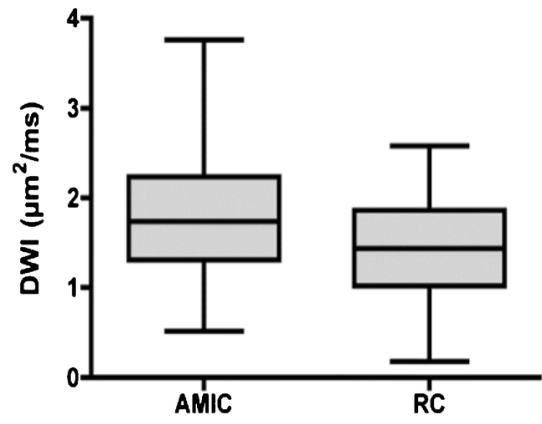

T2 Relaxation Time

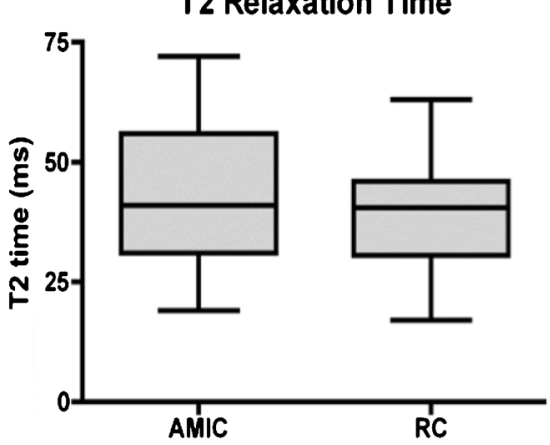


Fig. 4 Least-square means and standard errors of diffusion coefficients of the RT adjusted for the WORMS lesion score of the opposing tibial cartilage and RT size by tertiles of follow-up interval between surgery and MRI

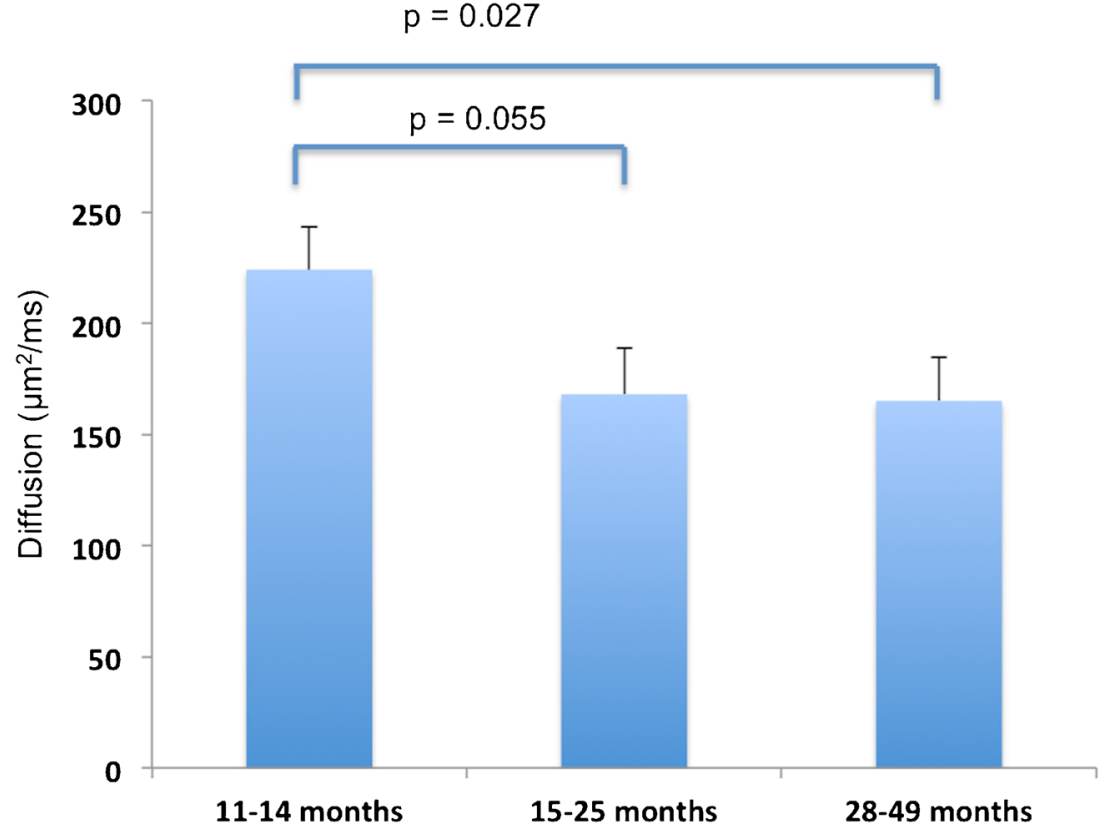

of fibrocartilage, while MACT RT shows more hyaline-like properties $[31,32]$. The similar T2 values of the AMIC-RT and reference cartilage may, indeed, indicate hyaline-like properties of the AMIC-RT, but in vivo studies on the histological properties of AMIC-RT, which might provide answers to this question, are still lacking.

Our finding that RT diffusion coefficients decreased with follow-up time is consistent with a study that investigated the development of diffusion coefficients of MFX-RT in knees [22], where a significant decrease in tissue diffusivity occurred in the first 24 months, and remained stable in the later followup. Our data suggest that the main down-slope of diffusivity has already occurred at 15 months, which may indicate increased density and matrix organization of RT as a result of tissue maturation.

There were limitations to the study that must be considered. First, only a small number of patients could be included, and this may have contributed to the high standard deviations. However, for one of the sequences, we found significant differences in the RT and control cartilage even in this small number of patients. Second, MRI data from this crosssectional study exist for only one point in time; thus we were not able to evaluate the longitudinal development of $\mathrm{T} 2$ values or diffusion coefficients within the RT. However, it would be very interesting to assess specific changes in the biochemical properties of the RT in further follow-up examinations, and this should be investigated in future longitudinal studies. With the pSSFP sequence, we applied a new technique for T2 mapping. To our knowledge, there is no published data regarding the clinical application of this technique. Thus, some uncertainty remains with respect to the comparability of the sequence used in our study with conventional techniques for
T2 mapping such as a multi-echo/spin-echo technique. Another study limitation is the fact that we could not correlate our findings with histological data, since a biopsy of the RT was not possible due to ethical considerations.

A strength of this study is the MRI protocol with identical resolution parameters of all 3D-acquired quantitative sequences, which allowed comparison between the different quantitative sequences.

In conclusion, our data indicate that the dwDESS is more sensitive to collagen network disorganization of AMIC-RT than quantitative T2 measures. dwDESS of RT also showed a cross-sectional association with the post-surgical follow-up interval. These findings render the technique a promising tool for the study of RT matrix changes in vivo during tissue maturation and longer follow-up periods. The reduced diffusivity in patients with longer follow-up times may indicate increased density and matrix organization of RT as a result of tissue maturation. The implications of this finding for the long-term outcome of AMIC-treated OCLs should be investigated in longitudinal studies.

Acknowledgments The scientific guarantor of this publication is Dr. Ueli Studler. The authors of this manuscript declare no relationships with any companies whose products or services may be related to the subject matter of the article. The authors state that this work has not received any funding. No complex statistical methods were necessary for this paper. Institutional Review Board approval was obtained. Written informed consent was obtained from all subjects (patients) in this study. Written informed consent was waived by the Institutional Review Board. Some study subjects or cohorts have been previously reported in Delayed gadolinium-enhanced MRI of cartilage of the ankle joint: Results after autologous matrix-induced chondrogenesis (AMIC)-aided reconstruction of osteochondral lesions of the talus. Wiewiorski et al. Clinical Radiology 6/2013. Methodology: prospective, observational, performed at one institution. 


\section{References}

1. O'Loughlin PF, Heyworth BE, Kennedy JG (2010) Current concepts in the diagnosis and treatment of osteochondral lesions of the ankle. Am J Sports Med 38:392-404

2. Brittberg M (2008) Autologous chondrocyte implantation-technique and long-term follow-up. Injury 39(Suppl 1):S40-S49

3. Benthien JP, Behrens P (2011) The treatment of chondral and osteochondral defects of the knee with autologous matrix-induced chondrogenesis (AMIC): method description and recent developments. Knee Surg Sports Traumatol Arthrosc 19:1316-1319

4. Wiewiorski M, Leumann A, Buettner O, Pagenstert G et al (2011) Autologous matrix-induced chondrogenesis aided reconstruction of a large focal osteochondral lesion of the talus. Arch Orthop Trauma Surg 131:293-296

5. Gille J, Schuseil E, Wimmer J, Gellissen J et al (2010) Mid-term results of Autologous Matrix-Induced Chondrogenesis for treatment of focal cartilage defects in the knee. Knee Surg Sports Traumatol Arthrose 18:1456-1464

6. Gille J, Meisner U, Ehlers EM, Müller A et al (2005) Migration pattern, morphology and viability of cells suspended in or sealed with fibrin glue: a histomorphologic study. Tissue Cell 37:339-348

7. Breinan HA, Martin SD, Hsu HP, Spector M (2000) Healing of canine articular cartilage defects treated with microfracture, a typeII collagen matrix, or cultured autologous chondrocytes. J Orthop Res 18:781-789

8. David-Vaudey E, Ghosh S, Ries M, Majumdar S (2004) T2 relaxation time measurements in osteoarthritis. Magn Reson Imaging 22: 673-682

9. Borthakur A, Shapiro EM, Beers J, Kudchodkar S et al (2000) Sensitivity of MRI to proteoglycan depletion in cartilage: comparison of sodium and proton MRI. Osteoarthritis Cartilage 8:288-293

10. Mlynárik V, Trattnig S, Huber M, Zembsch A, Imhof H (1999) The role of relaxation times in monitoring proteoglycan depletion in articular cartilage. J Magn Reson Imaging 10:497-502

11. Dunn TC, Lu Y, Jin H, Ries MD, Majumdar S (2004) T2 relaxation time of cartilage at MR imaging: comparison with severity of knee osteoarthritis. Radiology 232:592-598

12. Stahl R, Blumenkrantz G, Carballido-Gamio J, Zhao S et al (2007) MRI-derived T2 relaxation times and cartilage morphometry of the tibio-femoral joint in subjects with and without osteoarthritis during a 1-year follow-up. Osteoarthritis Cartilage 15:1225-1234

13. Li X, Benjamin Ma C, Link TM, Castillo DD et al (2007) In vivo $\mathrm{T}(1 \mathrm{rho})$ and $\mathrm{T}(2)$ mapping of articular cartilage in osteoarthritis of the knee using 3 T MRI. Osteoarthritis Cartilage 15:789-797

14. Blumenkrantz G, Lindsey CT, Dunn TC, Jin H et al (2004) A pilot, two-year longitudinal study of the interrelationship between trabecular bone and articular cartilage in the osteoarthritic knee. Osteoarthritis Cartilage 12:997-1005

15. Domayer SE, Kutscha-Lissberg F, Welsch G, Dorotka R et al (2008) T2 mapping in the knee after microfracture at $3.0 \mathrm{~T}$ : correlation of global T2 values and clinical outcome - preliminary results. Osteoarthritis Cartilage 16:903-908

16. Welsch GH, Mamisch TC, Domayer SE, Dorotka R et al (2008) Cartilage T2 assessment at 3-T MR imaging: in vivo differentiation of normal hyaline cartilage from reparative tissue after two cartilage repair procedures - initial experience. Radiology 247:154-161

17. Pearle AD, Warren RF, Rodeo SA (2005) Basic science of articular cartilage and osteoarthritis. Clin Sports Med 24:1-12

18. He B, Wu JP, Kirk TB, Carrino JA et al (2014) High-resolution measurements of the multilayer ultra-structure of articular cartilage and their translational potential. Arthritis Res Ther 16:205

19. Miller KL, Hargreaves BA, Gold GE, Pauly JM (2004) Steady-state diffusion-weighted imaging of in vivo knee cartilage. Magn Reson Med 51:394-398

20. Burstein D, Gray ML, Hartman AL, Gipe R, Foy BD (1993) Diffusion of small solutes in cartilage as measured by nuclear magnetic resonance (NMR) spectroscopy and imaging. J Orthop Res 11:465-478

21. Mlynárik V, Sulzbacher I, Bittšanský M, Fuiko R, Trattnig S (2003) Investigation of apparent diffusion constant as an indicator of early degenerative disease in articular cartilage. J Magn Reson Imaging 17: 440-444

22. Friedrich KM, Mamisch TC, Plank C, Langs G et al (2010) Diffusion-weighted imaging for the follow-up of patients after matrix-associated autologous chondrocyte transplantation. Eur J Radiol 73:622-628

23. Bieri O, Scheffler K, Welsch GH, Trattnig S et al (2011) Quantitative mapping of T2 using partial spoiling. Magn Reson Med 66:410-418

24. Bieri O, Ganter C, Scheffler K (2012) Quantitative in vivo diffusion imaging of cartilage using double echo steady-state free precession. Magn Reson Med 68:720-729

25. Marlovits S, Striessnig G, Resinger CT, Aldrian SM et al (2004) Definition of pertinent parameters for the evaluation of articular cartilage repair tissue with high-resolution magnetic resonance imaging. Eur J Radiol 52:310-319

26. Duc SR, Pfirrmann CW, Koch PP, Zanetti M, Hodler J (2008) Internal knee derangement assessed with 3-minute three-dimensional isovoxel true FISP MR sequence: preliminary study. Radiology 246: 526-535

27. Shepherd DE, Seedhom BB (1999) Thickness of human articular cartilage in joints of the lower limb. Ann Rheum Dis 58:27-34

28. Quirbach S, Trattnig S, Marlovits S, Zimmermann V et al (2009) Initial results of in vivo high-resolution morphological and biochemical cartilage imaging of patients after matrix-associated autologous chondrocyte transplantation (MACT) of the ankle. Skelet Radiol 38: 751-760

29. Marik W, Apprich S, Welsch GH, Mamisch TC, Trattnig S (2012) Biochemical evaluation of articular cartilage in patients with osteochondrosis dissecans by means of quantitative T2- and T2mapping at 3 T MRI: a feasibility study. Eur J Radiol 81:923-927

30. Apprich S, Trattnig S, Welsch GH, Noebauer-Huhmann IM et al (2012) Assessment of articular cartilage repair tissue after matrixassociated autologous chondrocyte transplantation or the microfracture technique in the ankle joint using diffusion-weighted imaging at 3 Tesla. Osteoarthritis Cartilage 20:703-711

31. Zheng MH, Willers C, Kirilak L, Yates P et al (2007) Matrix-induced autologous chondrocyte implantation (MACI): biological and histological assessment. Tissue Eng 13:737-746

32. Knutsen G, Engebretsen L, Ludvigsen TC, Drogset JO et al (2004) Autologous chondrocyte implantation compared with microfracture in the knee. A randomized trial. J Bone Joint Surg Am 86-A:455-464 